\title{
LA COSTURA Y EL BORDADO EN EL MUSEO DE ARTE NAÏF DE JAÉN
}

\author{
María del Carmen GILA MALO \\ Universidad de Granada (España) \\ cgilamalo@gmail.com
}

\begin{abstract}
NEEDLEWORK AND EMBROIDERY IN THE ART NAÏF MUSEUM OF JAÉN
Resumen: En el presente texto se considera que el bordado y la costura son técnicas artísticas que se aplican al dibujo y a la pintura, con igual categoría e importancia que otras técnicas, que han sido empleadas en los siglos XX y XXI, por todo tipo de artistas, tanto de renombre como menos conocidos, tanto hombres, como mujeres. Lo que concretamente se pretende es poner de manifiesto la situación del bordado y la costura como técnicas artísticas en el arte naif y mostrar a artistas que lo empleen expuestos en el museo "Manuel Moral". Y sobre todo, lo que se busca es darle una mayor importancia a esta técnica, que incluso cuenta con una sala en exclusiva dentro del museo.
\end{abstract}

Abstract: This text proposes that embroidery are considered an artistic technique that can be applied to drawing and painting; with the same importance as other techniques and that embroidery has been used by all kinds of artists, both male and female, over the course of the $20^{\text {th }}$ and 21 st century. The present research wants to show the present situation of embroidery as artistic technique in the Naîf Art and show artists who use it, whose work is in the "Manuel Moral" museum. And, finally, the present research goes one step further to give the real importance that this technique should have: it has its own hall in the museum.

Palabras clave: Bordado; Costura; Dibujo; Naif; Hilo

Embroidery; Needlework; Drawing; Naïf Thread 


\section{Introducción}

Con motivo de la reapertura del Palacio de Villardompardo tras su restauración, y por consiguiente del museo internacional de arte naif "Manuel Moral" de Jaén, y la lectura de la tesis doctoral de la autora, que investiga el uso del bordado y la costura en las artes plásticas, en la que por estar cerrado dicho museo no se ha podido investigar correctamente, se cree necesario poner de manifiesto el uso del bordado en el arte naif. Concretamente se va a revisar la situación del bordado y la costura como técnicas artísticas en el museo de arte naif de Jaén. Se quiere investigar y distinguir el bordado como técnica en el arte, y no como tradición renovada. Para ello, el presente texto se va a centrar en algunas obras realizadas con dichas técnicas, que se pueden encontrar en el museo internacional de arte naif "Manuel Moral".

Se va a empezar explicando brevemente el concepto de arte naif, siguiendo por una revisión del uso del bordado en el dibujo y la pintura, para terminar con el estudio de las obras bordadas expuestas en el museo de Jaén.

\section{El Arte Naif}

El arte naif es un tipo de arte cuya principal característica es que el autor de las obras no es un artista profesional, sino que es un aficionado o amateur, sin formación académica. Debido a esta falta de formación sus obras suelen ser muy ingenuas, como si hubiesen sido realizadas por niños (de ahí el nombre de naïf, que en francés significa ingenuo); no siguen los cánones, ni las proporciones y los problemas no son resueltos por las leyes establecidas, sino como cada artista ve más conveniente. Son dibujos y pinturas con colores muy saturados y planos; los dibujos son muy detallistas y suelen estar delineados; además suele primar una falta de perspectiva, tanto lineal, como aérea y una falta de volumen. En cambio, son obras extremadamente expresivas. En cuanto a los temas representados, suelen destacar la vida cotidiana, la religión o las costumbres; en ocasiones con una visión idílica del mundo, más que con un afán reivindicativo.

\section{El Bordado}

José Luis Espejo señala (Pilar Tobon et al., 2008: 18): "Cuando hablamos de arte textil estamos definiendo un complejo mundo de materiales que se han usado desde tiempos inmemoriales para la fabricación de telas". Esta tesis está suficientemente defendida en la actualidad, ya no hay discusión sobre la consideración del arte textil como un arte. En este texto se quiere dar un paso más y se plantea que el hilo puede no solo sustituir a los lápices y a los pinceles, sino que puede complementarse con ellos. El hilo y la tela pueden suponer unos materiales más que combinar con otras técnicas más ortodoxas.

\section{El bordado erudito y el bordado popular}

Antes de continuar con el bordado como técnica de dibujo, se quiere hacer una apreciación en cuanto a la clasificación del bordado. Una de las clasificaciones del bordado lo divide en dos grupos, el bordado popular y el bordado erudito, cuyas características son:

- Bordado erudito. Emplea materiales ricos, como telas de finas sedas, hilos de sedas y metálicos. Se suelen hacer con bastidor. El dibujo se tiene que dibujar en la tela porque no sigue la trama ni la urdimbre, es un bordado libre, esto explica la mayor extensión y variedad de los diseños. En cuanto a los motivos ornamentales, sigue las tendencias artísticas del momento y van cambiando según la época. Tienen más calidad artística cuando los diseños son del propio bordador y no un calco de una plantilla preexistente.

- Bordado popular. Emplea telas más gruesas, de algodón y lino e hilos de seda y algo- 
dón. Se suelen hacer a mano, siendo más fáciles de realizar. El dibujo suele seguir la trama y urdimbre de la tela y no necesita dibujarse en la misma porque se consigue contando hilos. En cuanto a los motivos ornamentales, la mayoría son versiones de unos mismos motivos.

\section{Bordado, costura y tejido}

Existe una clasificación bastante clara de dividir las técnicas de aguja, que las divide en tres grupos según su metodología de trabajo:

- Bordado. Están hechas con bordado las obras en las que el medio de dibujo es el hilo atravesando algún soporte, generalmente tela o papel. No se pueden entender las aplicaciones o appliqué fuera del bordado, ya que están íntimamente unidos y en muchos bordados se incluyen las aplicaciones.

- Costura. Como costura se va a entender a toda obra en la que se emplea el hilo como método de unión entre dos superficies, por ejemplo las almazuelas. Sin embargo, como se acaba de decir, excluyendo a los appliqué que se incluyen en el bordado.

- Tejido. Basándose en la definición de tejido de la R.A.E., se va a llamar tejido a la obra realizada con material hecho tejiendo o formada al entrelazar varios elementos con el empleo de una o varias agujas. Dentro de esta definición se encuentran el punto, el ganchillo y el tapiz.

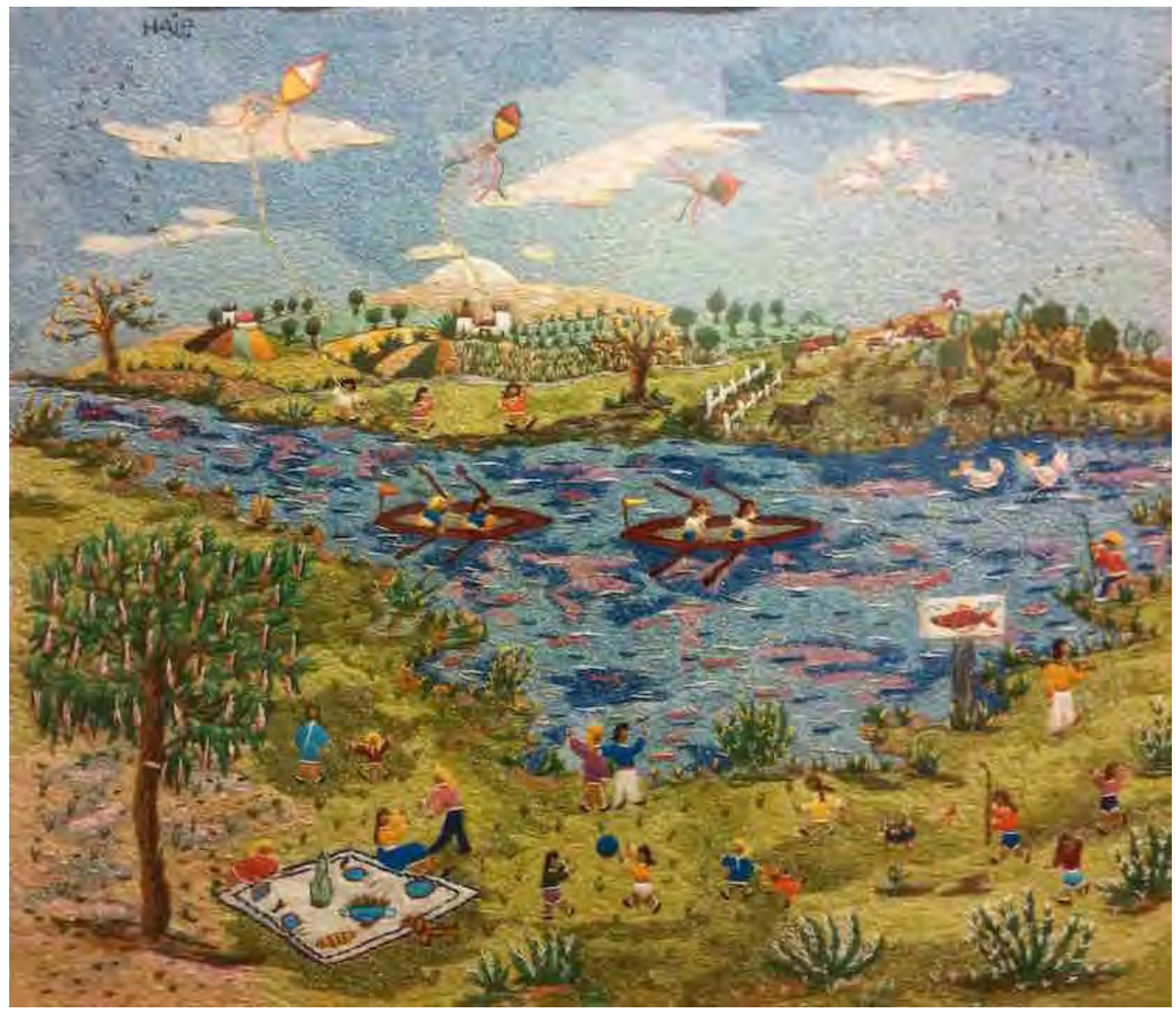

Figura 1. Isabel Pruna, Las Canoas, Barquita y Merienda, bordado sobre tela, $32 \times 37 \mathrm{~cm}$. Número inventario 3443. 


\section{Bordado, arte y lujo}

Si se tiene en cuenta la idea de que el bordado es un elemento decorativo sin ninguna utilidad práctica más allá de ser bello y si se tiene en cuenta la definición de lujo que ofrece Gilles Lipovetsky (Gilles Lipovetsky y Elyette Roux, 2004: 19) “el lujo es el ensueño, lo que embellece el decorado de la vida, la perfección hecha objeto por obra del genio humano" o la que ofrece Elyette Roux (Gilles Lipovetsky y Elyette Roux, 2004: 173) "el lujo remite al placer, al refinamiento, a la perfección, así como a la rareza y a la apreciación, costosa, de aquello que existe sin necesidad", entonces debemos considerar al bordado como un elemento de lujo, puesto que se ha empleado para embellecer y ornamentar ropa y elementos del hogar.

Lipovetsky dice que otras características que se le atribuyen al lujo tras la Revolución Industrial (y especialmente en la actualidad) son la innovación creativa, la manufactura impecable, y el valor estético. Por lo tanto, la verdadera esencia de un bordado, su auténtica importancia de existir, es la calidad de la factura y de la estética.

El arte en general, y el dibujo en particular, también son un elemento de lujo. Son la máxima expresión de la cultura de lo suntuario. El arte es el mayor nexo de unión que existe entre el lujo y la cultura.

En una sociedad en la que el lujo es valorado y es absolutamente moderno, ambos elementos (arte y bordado) tienen cabida. Con su unión, no se estaría desprestigiando ninguna de estas dos disciplinas, sino que se están equiparando en importancia.

\section{El bordado como dibujo}

Si ahondamos en los aspectos más conceptuales del bordado como dibujo, lo primero que se tiene que abordar son los elementos básicos del dibujo y la geometría: el punto, la línea y el plano.

El punto, del latín punctum, es el elemento primario de la expresión plástica, es la unidad más simple y sencilla en que se puede dividir una imagen; es la consecuencia del choque de un instrumento con la superficie. Cuando hablamos del bordado y la costura, una puntada es un sinónimo de punto y es la acción que se realiza cada vez que se hace un punto. En un dibujo realizado por ejemplo con tinta, podemos construir una imagen sin emplear un punto evidente. Se estaría aludiendo al término punto en su acepción más conceptual: un elemento básico sin forma ni dimensiones. En cambio, cuando se trabaja con el bordado es indispensable trabajar por puntos: sin puntos no sería bordado, podría ser un hilo pegado o un hilo unido.

La línea es una sucesión de puntos, es la historia de un punto en movimiento o son puntos en movimiento. La línea se produce al deslizar un útil por una superficie. Teniendo en cuenta esta definición una costura es la línea perfecta, ya que es una sucesión de puntadas o puntos formando una línea. La costura es un tipo de línea en el que es claramente evidente que se ha creado por una sucesión de puntos, de hecho se aprecia la historia de su creación.

El plano se define como un conjunto de líneas que se cortan o mantienen relaciones de paralelismo. Al igual que ocurre con los términos anteriores, es en la tela donde mejor se puede apreciar el concepto de plano. Una tela está formada por hilos paralelos y perpendiculares que se cortan, formando una trama, que produce una superficie plana.

Dentro del plano no aquí no se puede olvidar tratar el término textura, que según la RAE es la disposición y orden de los hilos en una tela. El diccionario de la Real Academia no hace alusión alguna a su significado de cualidad visual y táctil de una superficie, tan empleado en las artes plásticas. Por lo tanto también en el soporte comúnmente empleado en el bordado este término no es excluyente para con el bordado.

Por otro lado, si se estudia el bordado como método de dibujo desde un punto de vista más filosófico y relacionado con lo que dibujar significa, no hay que olvidar el libro Los 


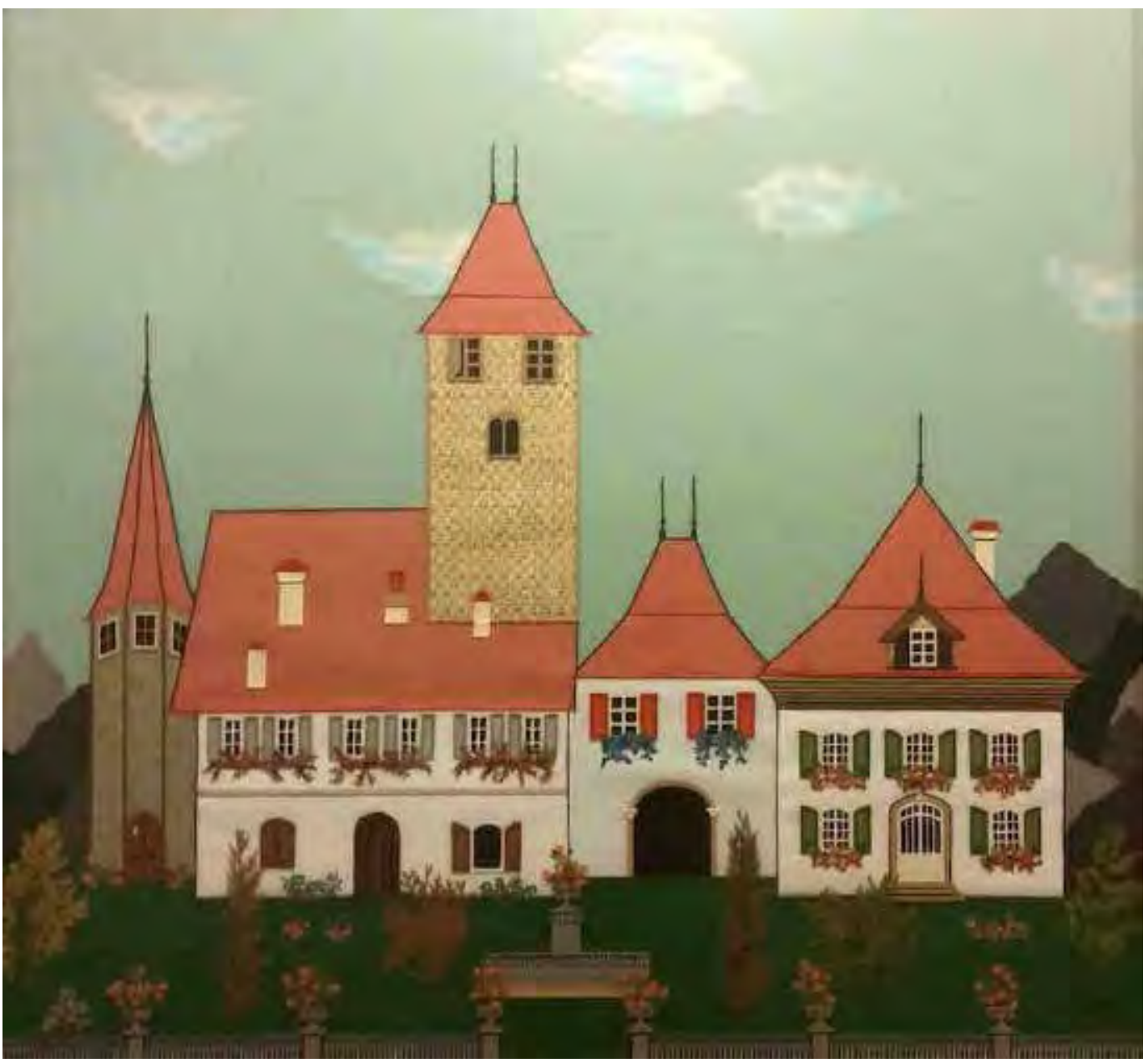

Figura 2. María del Carmen Gámez Lucena, Castillo de Esquiez, Suiza, bordado, costura y collage sobre tela, $100 \times 100 \mathrm{~cm}$. Número de inventario: 3331 .

Nombres del Dibujo, de Juan José Gómez Molina, Lino Cabezas Gelabert y Miguel Copón.

Miguel Copón aporta la siguiente definición de dibujo en el libro antes mencionado: "dibujar o delinear significa delimitar mediante sucesión de puntos el contorno o las formas significativas de un cuerpo" (Gómez Molina, Cabezas Gelabert y Copón, 2005: 510). Esta definición complementa a la perfección los párrafos anteriores, porque, como se ha dicho, la técnica del bordado se basa en la sucesión de puntos o puntadas.

Dice Juan José Gómez Molina que "lo fascinante del dibujo es la capacidad de dar cuenta tanto de aquello que nombra como la operación desde donde lo nombra" (Gómez Molina, Cabezas Gelabert y Copón, 2005: 87). El bordado es una operación para nombrar lo que se piensa, idea y siente que se caracteriza por permitir un medio de expresión diferente, alternativa y complementaria a otras técnicas más populares o comunes. Considerando al bordado como técnica artística se amplía el número de las mismas y permite nuevos modos de expresión, se aumenta el número de operaciones con las que nombrar la realidad.

Lino Cabezas comenta que "una obra de arte puede originarse de diferentes maneras: deliberada o accidental, impulsiva o dirigida, espontánea o resuelta metódicamente a partir del estudio y la reflexión” (Gómez Molina, Cabezas Gelabert y Copón, 2005: 253). Lo inte- 


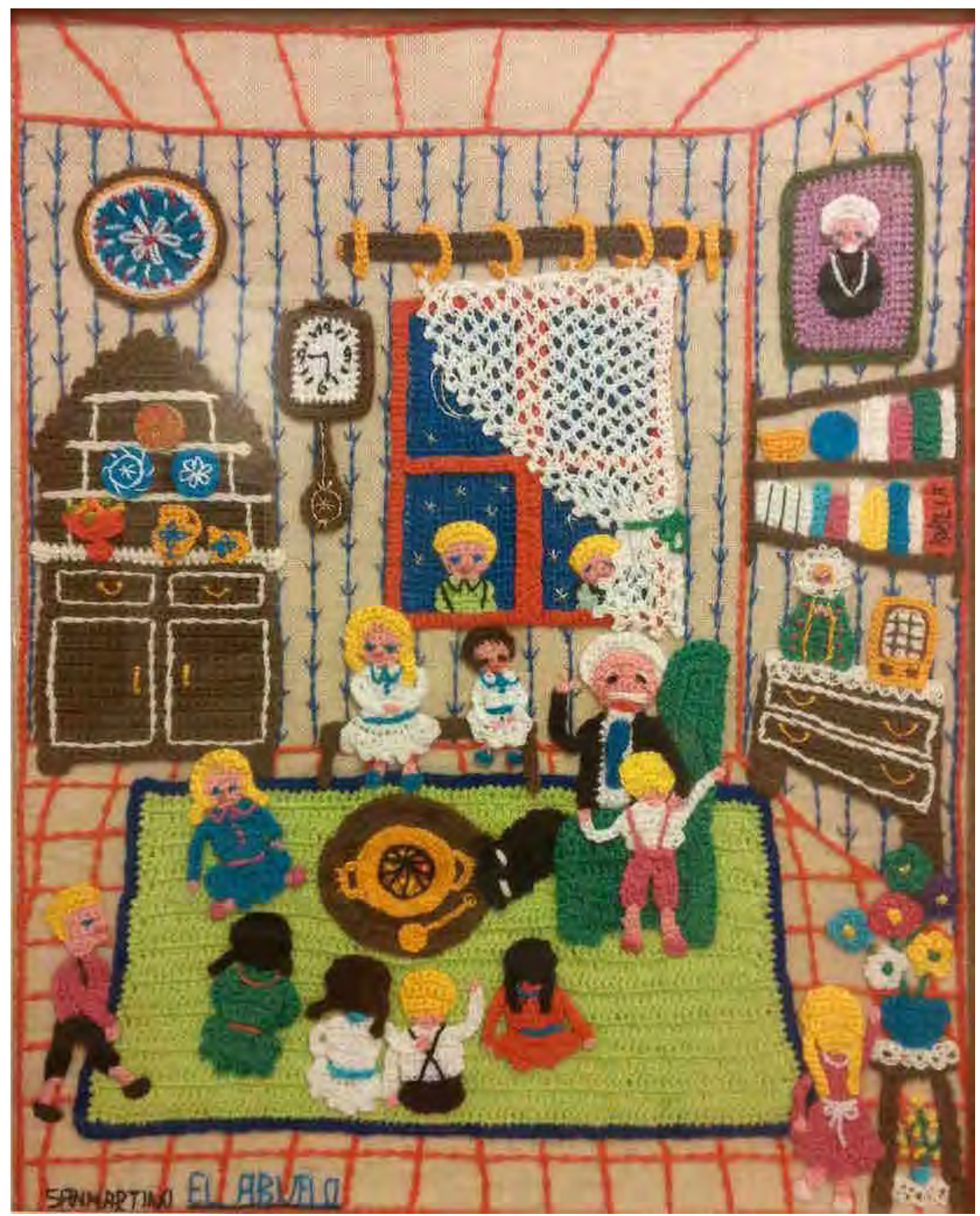

Figura 3. Lola Martino Moya, El Abuelo, bordado y tejido de ganchillo sobre tela, 37 x $30 \mathrm{~cm}$. Número de inventario: 3398. 
resante de este punto es que, a pesar de que el bordado pueda parecer una técnica deliberada, metódica y reflexiva, también permite el accidente, la impulsividad y la espontaneidad a partir de puntadas y costuras más libres y aleatorias. Es el estilo de los dibujos que hace la artista Ghada Amer, en los que se conjugan puntadas que limpiamente definen unos contornos, con los cabos sueltos de los hilos aportan confusión y desorden.

Marcel Duchamp decía que "cuanto más atractivo sensorial tuviese un cuadro (cuanto más animal fuese), mejor considerado era" (Duchamp, 1946, citado en Gómez Molina, Cabezas Gelabert y Copón, 2005: 261). Esta afirmación hace que no se olvide al bordado como un medio para hacer un dibujo o una pintura. Como se ha dicho, el bordado es una técnica muy táctil que permite ser sentida con las manos, lo que aumenta el atractivo sensorial de la obra frente a otra con una textura poco marcada.

\section{El Bordado en el Arte Naif}

Si se tiene en cuenta la división entre bordado erudito y bordado popular, se podría decir que los dibujos realizados mediante bordados que encontramos en el museo de arte naif pertenecen al primer grupo. Ya que cumplen varios de los requisitos: son dibujos que no siguen la trama y la urdimbre en sus diseños; los motivos no son los típicos de los bordados, copiados de una plantilla, sino que son dibujos personales y únicos. En lo que sí que difieren, pero que no es la característica más importante, es en la calidad de los materiales. En muchos de los dibujos expuestos en el museo no se emplean materiales nobles y ricos, lo que no les hace perder su estatus de obra de arte.

Como se ha dicho el bordado es un elemento suntuario, al igual que el dibujo o la pintura. El arte en general es un elemento de lujo. Por lo tanto, las obras expuestas en el museo de arte naif, se podrían considerar como un elemento de lujo, por su calidad técnica, por su estética y por las connotaciones que implican.

Si se tiene en cuenta la idea de Marcel Duchamp de que un cuadro es más considerado cuanto más atractivo sensorial tenga, los dibujos bordados del museo deben ser altamente considerados, ya que su atractivo sensorial se ve incrementado (como con todos los dibujos hechos mediante el bordado). A su atractivo visual hay que sumarle el atractivo táctil: el dibujo realizado con bordado o costura tiene un atractivo táctil superior a otras técnicas, ya que tiene diferentes volúmenes, sensaciones al tacto, texturas cálidas... El dibujo hecho mediante el bordado es un dibujo muy táctil. Además, al igual que la escultura, es muy apto para invidentes, porque mediante los relieves que deja el hilo sobre el soporte es fácil averiguar la forma y sentir el dibujo.

En el arte naif encontramos ejemplos de esta técnica, concretamente en el Museo de Arte Naif de Jaén, el único de España de este género, encontramos obras de artistas como:

Soledad San Martín Álvarez, alias Soledad Sarmartino
Letizia Arbereteta
María del Carmen Gámez Lucena
Lola Martino Moya, alias Lola Santartino
Marta Rodríguez Salmones
Nora Rojas Jara
Asunción Merino López
Isabel Pruna, alias Haip
Geneviere Ducroquet, alias Felicia
Silvia Echandi Ercila

A continuación se muestran tres obras, elegidas como representación de diferentes técnicas, por su calidad y creatividad frente a otras obras de dicho museo. También por ser obvias 
las técnicas con las que se realizan. Una con bordado, otra con costura y collage y la última con bordado y tejido, concretamente ganchillo:

Las Canoas, Barquita y Merienda, de Isabel Pruna (Figura 1), realizada con bordado sobre tela. Concretamente esta obra está realizada siguiendo la técnica de la pintura a la aguja; en la que los tonos se consiguen con puntadas de diferentes tonos de hilo. Es una variante del needlepoint, que en Europa "consistía en hacer copias exactas de obras de arte famosas empleando el bordado como técnica artística. Era una actividad muy de moda entre las clases altas que permitía a las obras de las mujeres entrar en los museos" (Gila Malo, 2014: 80-81). Este caso parece ser, que al igual que lo que hacía la artista granadina Trinidad Morcillo, que es una variante en la que no se copian obras, sino que se crean originales.

Castillo de Esquiez, Suiza, De María del Carmen Gámez Lucena (Figura 2), hecha con bordado, costura y collage sobre tela. Esta obra se caracteriza por el uso de diversos materiales y técnicas; ya que une tela, hilo, algodón, flores y hojas, con una factura de calidad.

El abuelo, de Lola Martino Moya (Figura 3), realizada con bordado y tejido de ganchillo sobre tela. Por último, esta obra une el bordado sobre tela, para las paredes y suelo, con las aplicaciones de formas realizadas con ganchillo de forma independiente, que posteriormente se han unido al soporte.

\section{Conclusiones}

Ante todo, como conclusión principal y debido a que el bordado es una técnica independiente (no un collage) y a que en el museo se le dedica una sala en exclusiva, se propone cambiar los letreros de las obras donde dice "collage de tejidos" por los términos correctos "bordado", "costura", "tejido".

El museo de arte naif ha permitido entrar la producción artística de las mujeres en el museo desde una doble vertiente: por ser artistas amateur y por el uso de una técnica diferente a la habitual.

Los bordados con los que se han realizado las obras que se exponen el museo pertenecen al grupo de bordados eruditos por su creatividad y originalidad y por su calidad técnica.

\section{Agradecimientos}

A mis padres, que me han apoyado moral y económicamente. A mis hermanos Ana y Francisco que han estado conmigo en los buenos y malos momentos. A mi abuela Carmen por enseñarme diferentes técnicas de bordado. A Leticia Alonso Morcillo, hija de Trinidad Morcillo, la valiosa información que me ha dado sobre su madre. A Asunción Jódar Miñarro, que me animó a escribir la tesis.

\section{Bibliografía}

BURGOS, Carmen de

1994 Moderno Tratado de Labores. Barcelona: Antonio J. Bastinos.

CAULFEILD, Sophia y SAWARD, Blanche

1882 The Dictionary of Needlework: an Encyclopadia of Artistic, Plain, and Fancy NeedleCERATO

2011 “Tipos de bordado" en Cenefas de Flores, Punto de Cruz, Cocina y Algo Más. http://cerato.wordpress.com/2011/01/13/tipos-de-bordados/ (29-08-2013).

DIPUTACIÓN PROVINCIAL DE JAÉN

s.f. $\quad$ "Museo Internacional de Arte Naïf Manuel Moral" en Cultura y Deportes. http://www. dipujaen.es/conoce-diputacion/areas-organismos-empresas/areaC/centro-cultural-palacio-villadompardo/arte-naif/ (22-10-2014). 
ECURED

s.f. "Arte Naif” en Historia de la Cultura. http://www.ecured.cu/index.php/Arte_Naif (22 -10-2014).

FATÁS, Guillermo y BORRÁS, Gonzalo M.

2000 Diccionario de Términos de Arte y Elementos de Arqueología, Heráldica y Numismática. Madrid: Alianza.

FLORIANO CUMBREÑO, Antonio C.

1942 El Bordado. Barcelona: Alberto Martín.

GILA MALO, María del Carmen

2014 Dibujar bordando. Aplicación del bordado al dibujo [Tesis doctoral. Orig. Inédito].

MARTÍ, Amparo y URBANO, Manuel

1998 Arte Naïf (catálogo del museo). Jaén: Diputación de Jaén e Instituto de Cultura.

REAL ACADEMIA ESPAÑOLA

2011 "Diccionario de la Lengua Española, Ed. XXII" en Real Academia Española. http://www. rae.es/rae.html (19-02-2013).

\section{3}

